\title{
Breaking logo's rule: Promoting social distancing to prevent COVID-19 spread by changing brand's logo
}

\author{
R.A. Siswanto \& J. Dolah \\ Universiti Sains Malaysia, Penang, Malaysia \\ I. Resmadi \\ Telkom University, Bandung, Indonesia
}

\begin{abstract}
In the current COVID-19 pandemic situation, a logo can convey social campaigns about social distancing. By delivering this social campaign, some brands have changed their logo shape without shifting their identity and recognizability. On the other hand, turning or twisting a logo could be seen as destroying it. However, this logo modification phenomenon shows that brands must be adaptive and flexible in responding to social situations to show their empathy and build positive perceptions and images in society. The method used in this study is the qualitative research method with a case study approach. The results of this paper aim at providing a picture of the creative process of making a logo that can respond to social phenomena. Logos can react to social phenomena, and go through a deconstruction of form, without having to lose its character and identity. This research shows that in the digital age the rigidity of a logo can be reduced by media, which allows a logo to be more flexible and fluid.
\end{abstract}

Keywords: logo design, branding, dynamic identity, campaign, COVID-19

\section{INTRODUCTION}

According to the World Health Organization (WHO), maintaining a distance of 2 meters between people could minimize the spreading of COVID-19 (Centers for Disease Control and Prevention 2020). This protocol, which later was known as "social distancing", became commonly discussed due to the global pandemic of COVID-19 that began in 2020. Social distancing itself is referred to as an action or measure taken to minimize the disease spread by reducing contact between large crowds. However, it's not only people who practice social distancing; several brands are also keeping the distance by tweaking their logos amid the coronavirus pandemic. Coca-Cola, McDonald's, Audi, and Volkswagen are some of the companies that have promoted "social distancing" into their logos by adding extra spaces in between in their logo elements.

These brands suggest people be aware of the current situation to keep the distance between people. This phenomenon escalates to become paradoxical and contradictory because logos should never be tweaked and must remain consistent in certain forms (Wheeler 2017). However, what is currently happening is that logos can then change shapes and become more fluid, flexible, and adaptive. Logos can respond to social phenomena, although it must break the rules. The rise of the number of brand logos that can react to the COVID-19 social phenomenon shows that in the current digital era, logos must be able to compromise and so be dynamic in responding to various issues, especially in social media. Brands must create empathy that is humane and show its alignment with the community while still paying attention to the part of visual identity while maintaining its distinctive and robust characteristics (Kotler et al. 2016).

Some previous literature shows that the logo must be dynamic in this digital era because one of them is the demand for digital media. In the sense of "Dynamic Identities," a company brand 
should not be static, but continue to grow in response to technological developments. Dynamic identity also emphasizes that changes in color, patterns, and shapes can change from external factors or factors outside the designer (Van Nes 2012). In addition to technological change factors and external factors, some changes in a brand's identity are also due to several things, including criticism, aesthetics, and communication ethics (Guida 2014). Brand identity also must be easy to adapt and flexible with certain content, situations, and contexts to respond to various things, and brand identity or visual identity must have the ability to respond and react to the surrounding environment (Felsing 2010). From some of the literature, it can be said that during the COVID-19 pandemic, a brand can respond to social phenomena through a campaign method related to changes in its visual identity (color and shape).

The novelty that would like to be displayed in this paper is how a social campaign's case study is distancing by deconstructing the logo. The logo has a distinctive and potent visual identity and can also be an exciting medium in conveying social campaigns without losing its distinctive and identical visual identity.

\section{METHODS}

This research is aimed at answering the question of how brands change and keep their identity when they change their logo in order to promote the social distancing recommendation while the identity of the brand still has to be recognizable. This is very important because according to Wheeler (2017), a consistent logo and recognizability is key for a successful branding. Therefore, it is expected that we could understand how far the logo distortion can be done. To answer the question, the qualitative research method is used in this paper with a case study approach. The case was chosen based on the validity of the campaign released by the brands, not fan-made or unofficial versions. The sample is determined based on the date of the release between April 1, 2020 to May 31,2020 as the WHO recommendation to prevent the COVID-19 spread was released.

The analysis that will be used is to do a matrix analysis by discussing each visual element. Then, each visual element will be examined along with the social context that influences the creative process of changing the logo. Several stages of analysis used in this paper are analyzing the visual form of each logo, and the related social context of the logo change. Atlas.Ti software is used as the tool at the analysis stage to minimize the human error factor.

\section{RESULTS AND DISCUSSION}

According to the results of the McDonald's, Volkswagen, Audi, and Coca Cola logo analysis, it can be said that the shape changes designed by these big companies are still part of a dynamic visual identity. These patterns are part of an interesting and unique campaign, because in creating positive perceptions and building empathy about COVID-19, companies can deconstruct logos, even though these changes clearly do not change their unique identities and characteristics (colors and shapes). So, the conclusions obtained from the visual analysis results above are that the logo can change more dynamically because it is influenced by external factors, and in this context, the COVID-19 pandemic. However, the change in a logo must also ultimately see changes or shifts in harmony without changing the distinctive essence of the visual identity. Dynamic logo changes are only possible if there is still a boundary line between the social context achieved and the change that still maintains its visual identity. From the analysis results obtained, a dynamic logo in response to a social phenomenon is very possible without having to radically change the entire logo, but what shape and color are the strongest visual identities by adding or reducing parts of the shape of a logo. Even so, the community must be able to perceive the logo's identity. 


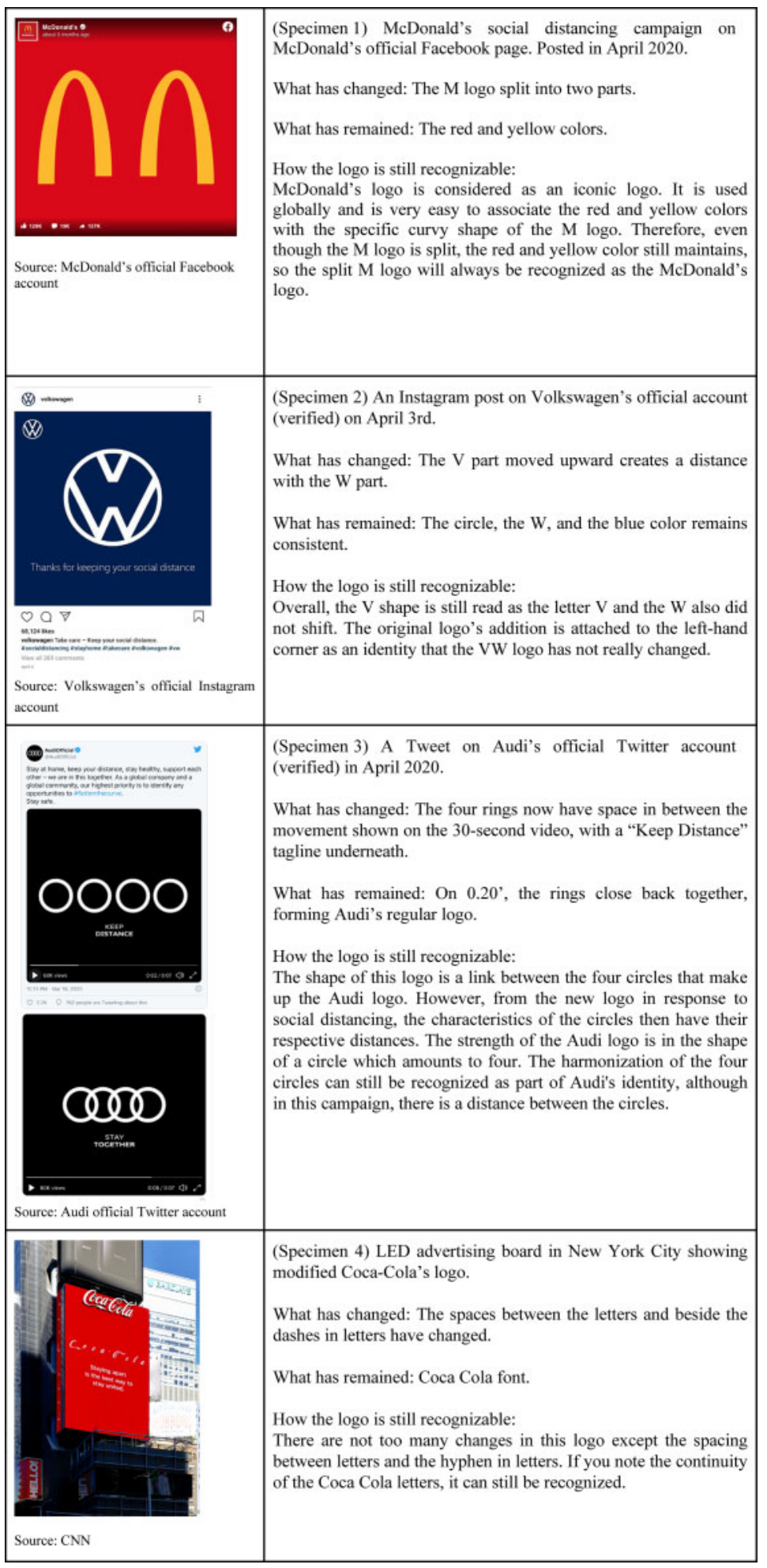

Figure 1. 


\section{CONCLUSIONS}

This research shows that the paradigm of a logo must be rigid and making it inflexible is increasingly irrelevant because, based on the case studies analyzed, indicating that the role of the logo is more than just a marker of a brand, but it can also be a media in showing empathy and concern for social situations and problems. As Kotler said, brands in the digital age must be more humane and behave, so that people will appreciate the brand, which will undoubtedly add to the brand's positive assumptions (Kotler et al. 2016). This research is still in the initial stages, where it is necessary to conduct studies that are more of studying the phenomenon or the deeper technical context. However, this research can show that in the digital age, the rigidity of a logo can be reduced by media, which allows a logo to be more flexible and fluid.

\section{REFERENCES}

Centers for Disease Control and Prevention (CDC). 2020. Social Distancing, Quarantine, and Isolation; CDC: Atlanta, GA.

Felsing, U. 2010. Dynamic identities in cultural and public contexts. Müller.

Guida, F.E. 2014. Dynamic identities for the Cultural Heritage. In Le vie dei Mercanti-XII Forum Internazionale di Studi (pp. 1113-1120). La Scuola di Pitagora Editrice.

Kotler, P., Kartajaya, H., and Setiawan, I. 2016. Marketing 4.0: Moving from traditional to digital. John Wiley \& Sons.

Van Nes, I. 2012. Dynamic Identities: How to create a living brand. BIS Publishers.

Wheeler, A. 2017. Designing brand identity: an essential guide for the whole branding team. John Wiley \& Sons. 